\title{
LAS VOCES DE UNA GENERACIÓN DE MAESTROS Y MAESTRAS QUE DEFENDÍAN UNA ESCUELA RENOVADA, VASCA Y POPULAR
}

\author{
Pauli Dávila Balsera \\ Universidad del País Vasco/Euskal Herriko Unibertsitatea UPV/EHU \\ pauli.davila@ehu.eus \\ Luis Maria Naya Garmendia \\ Universidad del País Vasco/Euskal Herriko Unibertsitatea UPV/EHU \\ luisma.naya@ehu.eus
}

\begin{abstract}
RESUMEN
El objetivo de este artículo es explicar la renovación pedagógica a partir de la recuperación de las voces del magisterio, como parte del patrimonio cultural de la escuela. Hemos estudiado una generación de maestros/as de más de 65 años, con más de 30 años de experiencia e implicados en la renovación pedagógica. Hemos realizado entrevistas, destacando la formación inicial, el ejercicio profesional y la formación permanente, que han sido analizadas mediante el programa NVivo. Entre los resultados obtenidos podemos resaltar: 1) la implicación en un fenómeno novedoso supuso un cambio de perspectivas pedagógicas teórico-prácticas, 2) esta actividad configuró una red de maestros y maestras que compartían una misma identidad profesional y diferencias en función del tipo de escuela y 3) el deseo de modernización pedagógica se vinculaba a la incorporación de la lengua vasca en las escuelas, en la defensa de una enseñanza popular.
\end{abstract}

Palabras clave: Renovación pedagógica. Historia oral. País Vasco.

\section{THE VOICES OF A GENERATION OF TEACHERS WHO DEFENDED A RENEWED, BASQUE AND POPULAR SCHOOL}

\begin{abstract}
The objective of this article is to explain the educational innovation from the recovery of the voices of the teaching profession, as part of the cultural heritage of the school. We have studied a generation of teachers over 65, with more than 30 years of experience and involved in educational innovation. We have conducted interviews, highlighting initial training, professional practice and lifelong learning, which have been analyzed by the Nvivo software. Among the results obtained we can highlight: 1) the involvement in a noew phenomenon supposed a change of theoretical-practical pedagogical perspectives, 2) this activity configured a network of teachers who shared the same professional identity and differences depending on the type of school and 3) the aim of the pedagogical modernization was linked to the incorporation of the Basque language in schools, in the defense of popular education.
\end{abstract}

Keywords: Educational Innovation. Oral history. Basque Country.

\section{AS VOZES DE UMA GERAÇÃO DE PROFESSORAS E PROFESSORES QUE DEFENDIAM UMA ESCOLA RENOVADA, BASCA E POPULAR}

\section{RESUMO}


Este artigo tem como objetivo explicar a inovação pedagógica a partir da recuperação das vozes da profissão docente, como parte do patrimônio cultural da escola. Para isso, estudamos uma geração de professoras e professores com mais de 65 anos de idade e com mais de 30 anos de experiência profissional em inovação pedagógica. O trabalho de campo foi feito através de entrevistas, prestando atenção na formação inicial, na prática profissional e também, na formação contínua. A análise das entrevistas foi feita por meio do programa NVivo. Entre os resultados podemos sublinhar os seguintes: 1) a implicação em um fenômeno inédito que supôs uma mudança de perspectivas pedagógicas teórico-práticas; 2) essa atividade configurou uma rede de professoras e professores que compartilhavam uma mesma identidade profissional e, da mesma forma, tinham diferenças dependendo do tipo de escola onde eles tinham trabalhado, 3) o desejo de modernização pedagógica estava ligado à incorporação da língua basca nas escolas em defesa do ensino popular

Palavras-chave: Inovação Pedagógica, História Oral, País Basco.

\section{LES VOIX D'UNE GENERATION D'ENSEIGNANTS QUI ONT DEFENDU UNE ECOLE RENOUVELEE, BASQUE ET POPULAIRE}

\section{RÉSUMÉ}

L'objectif de cet article est d'expliquer l'innovation pédagogique à partir de la récupération des voix du corps enseignant, comme partie du patrimoine culturel de l'école. Nous avons étudié une génération d'enseignants de plus de 65 ans, ayant plus de 30 ans d'expérience et impliqués dans l'innovation pédagogique. Nous avons mené des entretiens, mettant en avant la formation initiale, les pratiques professionnelles et la formation continue, analysées avec le programme NVivo. Parmi les résultats obtenus, on peut souligner: 1) l'implication dans un phénomène nouveau a eu comme conséquence un changement de perspectives pédagogiques théoricopratiques, 2) cette activité a configuré un réseau d'enseignants partageant la même identité professionnelle et différences selon le type d'école et 3) le désir de modernisation pédagogique était lié à l'incorporation de la langue basque dans les écoles, dans le cadre de la défense de l'enseignement populaire.

Mots-clès: Innovation pédagogique. Histoire orale. Pays Basque.

\section{INTRODUCCION}

La recuperación de las voces de los protagonistas en un determinado fenómeno educativo es fundamental para explicar históricamente el papel y la formación de las subjetividades. En esta colaboración estudiamos: 1) un fenómeno reciente en la historia de la formación del profesorado español, como es la renovación o innovación educativa; 2) la construcción de la identidad profesional y la conformación de las subjetividades de los maestros y maestras implicados y 3) el valor patrimonial de las voces del profesorado.

En los últimos años de la dictadura franquista y hasta la actualidad, tanto por parte de las autoridades educativas, como por parte del propio profesorado se llevó a cabo un proceso de reforma e innovación en la enseñanza. El papel principal recayó sobre un grupo de maestras 
y maestros renovadores que desarrolló una serie de estrategias de innovación pedagógica que lograron modernizar el sistema educativo.

El objetivo de este artículo es explicar este fenómeno a partir de la participación e implicación personal del propio magisterio, que formó una generación que compartía los mismos ideales pedagógicos y políticos. La recuperación de sus experiencias y expectativas profesionales conforma un conjunto patrimonial donde sus voces adquieren gran importancia en la conformación de la subjetividad y la identidad profesional. Para ello, hemos estudiado una generación de maestros/as que, en la actualidad, cuentan entre 65 y 75 o más años y que ha ejercido la profesión por más de 30 años en las escuelas del País Vasco, con implicación en la renovación pedagógica. Se trata de una generación de maestros/as renovadores. El caso del País Vasco es similar al resto de España, con el único elemento diferencial de la lengua vasca.

La metodología cualitativa aplicada en esta investigación se basa en un conjunto de entrevistas en profundidad realizadas a una serie de maestros/as que cumplen esas características. El foco de las entrevistas ha estado centrado en el ámbito de la renovación pedagógica, destacando tres momentos en la vida en su itinerario profesional: la formación inicial, el ejercicio profesional y la formación permanente. Esta metodología se diferencia de las historias de vida o de los relatos biográficos ya que son meros testimonios de sus experiencias personales en un ámbito concreto de la actividad profesional. Para analizar el contenido de las entrevistas hemos utilizado el programa para análisis de datos cualitativos NVivo.

Entre los resultados obtenidos podemos resaltar: 1) que la implicación en un fenómeno novedoso y de renovación pedagógica supuso un cambio de perspectivas pedagógicas tanto en la teoría como en la práctica, 2) que esta actividad configuró una red de maestros y maestras que compartían una misma identidad profesional y diversas subjetividades en función del tipo de escuela y 3) que, en el caso del País Vasco, el deseo de modernización pedagógica se vinculaba a la incorporación de la lengua vasca en las escuelas y la demanda de una escuela popular.

\section{EL PROFESORADO, LA RENOVACIÓN PEDAGÓGICA Y EL CONCEPTO DE GENERACIÓN}

El concepto de generación surge en los años 20 del siglo pasado (Marías, 1949). No obstante, la recuperación actual que se intenta hacer de este concepto se inscribe dentro de los estudios sobre las experiencias vitales, la descripción de las edades de la vida y su utilidad en 
los desafíos intergeneracionales. Lo cierto es que algunos investigadores plantean problemas sobre su uso en las ciencias sociales ya que es muy complejo aplicar las categorías del pasado para analizar la realidad actual (CABALLERO; BAIGORRI, 2013). La significación de una duración de la vida de los individuos en un lapso de tiempo determinado (de quince o veinte años) y su ubicación en la historia, compartiendo determinados acontecimientos históricos, creencias y comportamientos comunes $\mathrm{y}$, posiblemente, vinculados a un sentimiento de pertenencia a un grupo, parecen ser elementos suficientes para hablar de generación. A partir de aquí pasar a construir una identidad generacional podría ser una opción y, en algunos momentos, los individuos pueden llegar a referirse a ellos mismos con un sentimiento de pertenencia identitario que evita mayores matices individuales de las propias experiencias. En todo caso las generaciones, tanto en los ámbitos literarios, culturales o sociales demandan constantemente la construcción de un estereotipo para ser reconocidas y de algunos elementos claves que permitan reconocerse a sí mismas y ser identificadas por los demás.

La aplicación del concepto de generación al estudio del magisterio aporta un valor nada despreciable para conocer las mentalidades, las formas de trabajar, de asociarse y de compartir teorías, prácticas e innovaciones educativas y didácticas. De esta manera, las biografías personales, las historias de vida de cada uno de los maestros y maestras se transforman en una biografía coral, donde es más fácil comprender los matices y aportaciones del magisterio de una manera global, destacando las aportaciones individuales, las características y rasgos compartidos más allá de la biografía personal.

Por otra parte, hay que señalar que la bibliografía sobre el itinerario formativo del magisterio desde la perspectiva histórica, se corresponde con el análisis de la formación inicial en las escuelas normales o de magisterio y también con el asociacionismo profesional, así como con los movimientos de renovación pedagógica (PERICACHO, 2014; PINTO, 2016). No obstante, resultan del mayor interés los estudios que se están realizando, en general por investigadores/as con una visión interdisciplinar, de lo que podíamos denominar la formación de la identidad profesional, entendiéndola como la forma en que los profesionales se definen a sí mismos y a los otros. Se trata de una construcción del sí mismo profesional que evoluciona a lo largo de la carrera docente y que puede verse influida por la escuela, las reformas, los contextos políticos, etc. (LARSKY, 2005). Así pues, en la amplia bibliografía se recogen diversos acercamientos sobre el estudio del profesorado, tanto frente al cambio social, como a la formación inicial y permanente o a la construcción del conocimiento profesional-docente (GIL, 1996). Sin embargo, trabajos más recientes sobre identidad profesional (LEITE, 2012; 
LOPEZ et al., 2013) se centran en la investigación biográfica como la técnica más apropiada para la construcción de la identidad profesional o la cultura del profesorado.

Por lo que respecta al estudio de la renovación pedagógica en el País Vasco, ésta tiene una larga tradición (DÁVILA, 2005 y 2018). No obstante, todavía no se han realizado estudios en profundidad sobre las aportaciones de algunas asociaciones de profesores como Adarra (BIOTA; ZABALETA, 2018) o Gordailu (DELGADO, 2016), que fueron fundamentales para entender este fenómeno, además de los programas realizados desde la propia Consejería de Educación del Gobierno Vasco y de los Centros de Profesorado que existieron, con denominaciones diversas, desde 1984.

\section{METODOLOGÍA}

El estudio de esta generación de maestros y maestras nos va a permitir conocer mejor la dinámica interna y los itinerarios seguidos por el profesorado en la última parte del siglo XX en el País Vasco. Para ello partimos de la hipótesis de que el profesorado implicado ha sido un agente relevante en este proceso de renovación pedagógica. A través del análisis de esta generación constataremos las continuidades y rupturas, los agentes colaboradores, la implicación personal y los resultados de este proceso. Algunos de los testimonios, a pesar de haber ejercido como maestros, los hemos analizado como si fueran voces de expertos, debido a que, a lo largo de su trayectoria profesional, han ocupado diversos cargos en la administración educativa o como representantes de asociaciones profesionales de magisterio.

El objetivo general es analizar, a través de testimonios orales de esta generación, sus itinerarios formativos y profesionales. Para ello, el conocimiento de las voces de los protagonistas es importante, en tanto que marca el nivel de subjetividad y de construcción narrativa de los profesionales de la educación frente a las reformas educativas. Con este objetivo hemos realizado veintisiete entrevistas a maestros (10) y maestras (17) que, en los últimos cincuenta años, han participado de forma activa en este proceso.

Por lo tanto, se trata de un objetivo que, desde la perspectiva teórica y metodológica, está relacionado con un nuevo campo abierto en el estudio del profesorado, la profesionalidad docente a través de las historias de vida. No obstante, hay que señalar que la opción metodológica que hemos desarrollado en esta investigación persigue utilizar los relatos de vida del magisterio como elemento sustancial de la implicación personal en su desarrollo profesional. 
En este campo existe una amplia bibliográfica (SANCHO, 2011 y 2014; BOLÍVAR, 2005 y 2014; BOLÍVAR, DOMINGO, FERNÁNDEZ, 2001; IMBERNÓN, 2005; NOVOA, 1992; RIFÁ, 2005; ESCUDERO, GOMEZ, 2006; VIÑAO, 2009; ZUFIAURRE, 2007). En la tipología provisional establecida por Viñao (1999), la investigación que hemos desarrollado podría incluirse en la modalidad de la entrevista autobiográfica, que es otra modalidad textual más de este género, ya que ha sido grabada, aunque no esté impresa, que es el objeto de clasificación de este autor.

Para realizar el análisis de nuestros testimonios hemos seguido los siguiente pasos, de acuerdo con las aportaciones metodológicas al uso en este tipo de investigación. En primer lugar, determinamos el colectivo objeto de análisis. Así, nos pareció pertinente dividir este colectivo en tres generaciones de maestros/as que han desarrollado su actividad profesional en los últimos cincuenta años: una primera generación que se corresponde con un formación inicial recibida en el periodo anterior a la Ley General de Educación (1970); la segunda generación formada por maestros y maestras que desarrollaron su actividad laboral con la puesta en marcha de esta ley, que supuso una modernización del sistema educativo español, y la tercera se corresponde a la etapa de la Ley de Orgenación General del Sistema Educativo - LOGSE (1990), que también ha supuesto cambios profundos en dicho sistema. No obstante, al recoger los testimonios pudimos apreciar que, en este caso, la realidad se imponía y que estas tres generaciones compartían un mismo "humus", como hemos planteado previamente, por lo que nos pareció más pertinente tomarlos como una única generación que abarcaba desde comienzo de los setenta hasta los años noventa, donde ya se registran unos cambios importantes dentro de la formación inicial y permanente del profesorado. De esta manera, la tercera generación perdía su valor frente a los retos de la renovación pedagógica, debido a su normalización institucional. Hay que señalar que, en el País Vasco, la influencia de la LOGSE, siendo importante, se verá ensombrecida por la Ley de la Escuela Pública Vasca de 1993, que marcó un hito importante en la adscripción de las ikastolas ${ }^{1}$ a través de la obligada elección entre red pública o privada. En este contexto de cambios continuos, el profesorado se verá directamente interpelado a tomar decisiones de calado en su trayectoria profesional. Por otra parte, en la elección del colectivo también hemos tenido en cuenta la variable de género, no solamente por la feminización creciente de la profesión docente, sino porque en algunos casos su presencia es casi hegemónica. La opción elegida para la recogida de la información ha sido la realización de

\footnotetext{
${ }^{1}$ Centros escolares que enseñan únicamente en lengua vasca.
} 
una entrevista con un cuestionario semiestructurado, sobre los tres temas objeto de la investigación: la formación incial, la formación permanente y la actividad profesional.

Una vez transcritas las entrevistas, el equipo investigador comenzó la tarea de análisis y categorización de las mismas, utilizando el software Nvivo. Las categorías que se obtuvieron y su descripción se recogen en la tabla 1 y se corresponden a las tres dimensiones que han servido de base para la indagación sobre la formación y la actividad pedagógica.

Cuadro 1.- Descripción de las categorías correspondientes a la formación inicial y continua y al ejercicio profesional.

\begin{tabular}{|l|l|l|}
\hline Formación inicial & Ejercicio profesional & Formación continua \\
\hline $\begin{array}{l}\text { Razón por la que decidió } \\
\text { ser maestro o maestra }\end{array}$ & $\begin{array}{l}\text { Iniciación profesional en } \\
\text { el ámbito escolar }\end{array}$ & $\begin{array}{l}\text { Modelos de escuela y formación } \\
\text { conocidos }\end{array}$ \\
\hline $\begin{array}{l}\text { Características principales } \\
\text { de la formación inicial }\end{array}$ & Actividad profesional & $\begin{array}{l}\text { Actualización docente y su reflejo } \\
\text { en la actividad profesional }\end{array}$ \\
\hline $\begin{array}{l}\text { Las prácticas en el } \\
\text { periodo de formación }\end{array}$ & Carencias formativas & $\begin{array}{l}\text { Asociación grupo u organización } \\
\text { que ha influido en la formación } \\
\text { permanente }\end{array}$ \\
\hline $\begin{array}{l}\text { Valoración de la } \\
\text { formación inicial }\end{array}$ & $\begin{array}{l}\text { Tipos de apoyos } \\
\text { recibidos en la actividad } \\
\text { docente }\end{array}$ & $\begin{array}{l}\text { Participación activa en movimiento } \\
\text { de renovación pedagógica }\end{array}$ \\
\hline
\end{tabular}

Fuente: Elaboración propia.

\section{LAS VOCES DE LOS MAESTROS/AS RENOVADORES VASCOS}

El núcleo central de este trabajo ha sido recoger las voces de los maestros/as anónimos en su quehacer profesional y formativo. La voz de estos testimonios se diluye en un itinerario profesional y biográfico, dentro de un contexto social y educativo de transición política, en una etapa relevando de la reciente historia del Estado Español y el País Vasco.

La formación inicial es un momento clave en la vida de los maestros/as, ya que es cuando descubren las posibilidades de cambiar la escuela. El ejercicio profesional, en segundo lugar, es el momento en el que entran en contacto con un grupo de profesores con el que compartirían estos deseos de cambio en un contexto social y político y, finalmente, la formación permanente les permitió ir descubriendo nuevos discursos y prácticas para mantener viva la llama de la renovación pedagógica. En los siguientes epígrafes señalamos algunos resultados sobresalientes de cada uno de los momentos señalados.

Por otra parte, no podemos dejar de resaltar el valor patrimonial de las voces del magisterio, al igual que el de todas las personas involucradas en los fenómenos educativos: 
inspectores, alumnado, políticos, etc. ${ }^{2}$. Contar con unos documentos sonoros y visuales en los que se recogen los testimonios del magisterio amplía el concepto de patrimonio históricoeducativo y aporta experiencias y vivencias personales de indudable interés. En este sentido, estas grabaciones formarán parte del patrimonio recogido y expuesto en el Museo de la Educación de la Universidad del País Vasco, una de cuyas secciones está reservada a la formación del profesorado y la renovación pedagógica. Con ello se pretende acercar al público visitante las propias voces de los protagonistas y sujetos de la historia de la educación. La narración museística queda complementada con los registros de estas entrevistas, ya que, a través de medios audiovisuales, el público visitante puede escuchar y ver estos testimonios a través de la voz directa de sus protagonistas. En este sentido, esta aportación audiovisual es de una gran potencialidad al poner en contacto al público con el discurso histórico-educativo que se desarrolla. Además de estos testimonios, logrados a través de esta investigación, el público visitante tiene acceso, a través del Centro de Documentación del Museo a otra serie de entrevistas a maestros y maestras y exalumnado en las que se narran sus propias experiencias, estas narraciones proceden de un repositorio que está en acceso público ${ }^{3}$.

\section{El magisterio vasco ante la formación inicial}

La formación inicial del magisterio ha variado mucho, sobre todo a partir de la creación de un cuerpo de funcionarios del magisterio a principios del siglo XX. No obstante, todavía podemos encontrar textos muy actuales en los que se vuelve a reflexionar sobre el oficio de maestro en una sociedad cada vez más compleja (IMBERNÓN, 2017). Esta actualidad se debe, sobre todo, a que cada sociedad se plantea el tipo de formación que debe poseer el magisterio para atender las necesidades sociales y educativas, que son cada vez más cambiantes.

Al margen de los programas políticos sobre el acceso a los estudios del magisterio, existe un elemento reiterativo clave en la elección de esta profesión: la motivación para elegir el magisterio como profesión. Así, además de una feminización de la profesión cada vez más constatable, se aprecian expresiones como: "me gusta tratar con los niños" o "elijo la profesión por vocación" (ORTEGA, 1999). En nuestro trabajo hemos podido recoger que estas expresiones continúan vigentes. En cambio, en una obra reciente, se recoge la voz del profesorado con más de 30 años de experiencia que, a la misma pregunta sobre la motivación

\footnotetext{
${ }^{2}$ En el caso del País Vasco se puede consultar la Web https://ahotsak.eus/spanish/ en la que se pueden encontrar variados testimonios sobre la vida cotidiana, la infancia, la situación del euskara o el tránsito por las escuelas.

${ }^{3}$ Accesible en: www.ahotsak.net Recuperado en: 28 de octubre de 2018.
} 
para ser profesor/a, responde de manera más diversificada: "yo no tuve vocación, diferentes circunstancias me llevaron a estudiar magisterio"; "en principio, una idea de servicio. Alguno de mis profesores de bachillerato fue el 'causante' de tal decisión"; "no tenía ningún interés. [Eran] Estudios fáciles para alguien desmotivado"; "desconozco si la educación es cuestión de vocación, pero en mi caso reconozco, con toda honestidad, que no he sentido esa inclinación o tendencia interior" (IMBERNÓN, 2017). Como vemos, estos testimonios contrastan con la tendencia tradicional y muestran cierto desencanto creciente por la profesión docente.

Cuando nuestros testimonios se refieren a la razón por la que decidieron ser maestros/as existe cierta unanimidad al referirse a la preocupación por la situación política y la pérdida del euskera $^{4}$, por lo que su elección profesional es más contextual que personal. En cambio, cuando se refieren a los aspectos más propiamente educativos permanece la imagen de una escuela franquista, posiblemente vivida en la niñez, que es rechazada. Tanto una cuestión como la otra están llenas de matices y se aprecia que, en algunos testimonios, la opción por dedicarse a ser maestro/a está condicionada por estas cuestiones contextuales. El deseo de cambiar la escuela es evidente y, con ello, contribuir a un movimiento social que estaba luchando por la recuperación de la lengua y la cultura vascas, en un contexto de represión lingüística e inmigración foránea.

El contexto político viene a ser determinante en la decisión de dedicarse a la profesión docente. Algunos de los testimonios llegan a revivir la época (los años 80 y principios de los 90) de manera que el relato se convierte en una especie de crónica política de hechos y acontecimientos donde las interrelaciones políticas y la acción de muchos protagonistas de la época se presentan como los culpables o líderes de la toma de ciertas decisiones. De cualquier manera, la idea de la escuela franquista permanece inalterable, a pesar de que se represente más como un recuerdo de su propia escolarización, en algunos casos, que como una experiencia vivida en su formación como maestros/as. Estos relatos, cobran más relieve cuando el testimonio ocupa o ha ocupado algún cargo en alguna asociación, sobre todo en lo referente a convertir las ikastolas en centros públicos (Testimonio IE; Maestro, Ikastola). Muchas de las explicaciones que se transmiten tienen que ver con el proyecto educativo, en este caso vinculado a las ikastolas (EUSKAL HERRIKO IKASTOLAK; EUSKALTZAINDIA, 2011), en un contexto donde la Consejería de Educación estaba en manos de los socialistas. En 1993, con la Ley de la Escuela Pública Vasca culminará con el proceso de publificación de las ikastolas. Esta ley del Gobierno Autónomo Vasco cierra una discusión muy presente entre el magisterio

\footnotetext{
${ }^{4}$ Euskara o euskera: lengua vasca.
} 
y la propia sociedad vasca, como era la de mantener un sistema escolar con tres redes educativas: pública, privada e ikastolas, ya que, finalmente, las ikastolas tuvieron que decidirse su adscripción a la red pública o mantenerse como centros privados, aun recibiendo financiación pública para su funcionamiento. Hay que tener presente que las ikastolas han construido, desde su nacimiento, una identidad apegada al territorio y a la defensa de la lengua y la cultura vascas, característica que no se apreciaba ni en la red pública ni en la red privada religiosa. En el imaginario colectivo de muchas familias y maestros y maestras las ikastolas debían haberse convertido en la "verdadera" red pública escolar en el País Vasco. Otros testimonios ponen de manifiesta el "dolor" sufrido por el proceso vivido en 1993 con la publificación de las ikastolas, así uno de los testimonios afirma que "el proceso fue muy doloroso [...] Como pueblo aquel proceso nos hizo mucho daño" (Testimonio JMA; Maestro, Ikastola). La división creada por este proceso todavía está presente en muchas ikastolas. Este testimonio es una muestra del conflicto vivido por los propios protagonistas al ver que la única alternativa era aceptar el mandato legal. A partir de este momento muchas ikastolas se agruparon en una asociación de escuelas privadas que defienden la ikastola como el modelo del sistema de enseñanza vasco.

Hay que tener en cuenta que hasta la creación del Gobierno Vasco en 1979, la red pública escolarizaba casi exclusivamente en castellano, impartiendo el euskara como una asignatura, en cambio las ikastolas, desde su nacimiento, escolarizaban casi exclusivamente en euskara. En este sentido, muchos maestros y maestras eran conscientes de que su labor era euskaldunizar la escuela pública con el fin de que el euskara no fuera un patrimonio exclusivo de las ikastolas. Por lo tanto, la situación de pérdida del euskera es contemplada desde una doble perspectiva. Por una parte, afirmando que el euskera podría sobrevivir a través de su presencia en las ikastolas y, por otra, constatando que no podía limitarse únicamente a la presencia en ellas: "Nosotros pensábamos que el euskara no podía quedarse en las ikastolas. De otra manera caeríamos en un reduccionismo o gueto, el euskara debería extenderse a toda la sociedad" (Testimonio UB; Experta, Red pública). Para ello, las referencias a la realidad social eran el recurso habitual, ya que en los años 80 la población inmigrante era una parte importante del panorama demográfico en el País Vasco.

Otros testimonios muestran una opción más personal en cuanto a la decisión de dedicarse a la profesión docente, sin olvidarnos de este contexto social, político y educativo. Nos referimos a Neli Zaitegi, actualmente presidenta del Consejo Escolar de Euskadi, quien recuerda que fue una de sus maestras la que estimuló a tomar esta decisión ya que se trataba de una mujer "terriblemente innovadora" (Testimonio NZ; Maestra, Escuela pública) o a Joxe Maria Auzmendi, director de la revista Hik Hasi, quien destaca como elemento clave para 
dedicarse al magisterio la "pasión por la educación de los niños" (Testimonio JMA; Maestro, Ikastola), así como la situación sociopolítica.

En cuanto a la formación inicial recibida, la visión general suele ser bastante crítica, bien por su carácter autoritario o bien por tratarse de un tipo de escuela cuyos contenidos eran muy superficiales y que no sintonizaban con los deseos de cambio que se comenzaban a vivir en el País Vasco en los años 70. Asimismo, la formación recibida en las prácticas es altamente criticada porque no tenía ninguna conexión con la realidad. En algunos casos se resalta la creación de grupos alternativos que significaron una experiencia interesante de cara a la propia formación, al margen de la escuela oficial o bien la búsqueda de otras prácticas pedagógicas. En algunos casos se alude a maestras en ejercicio que sirvieron como modelos para el cambio en las metodologías didácticas. También resulta interesante ver cómo algunos testimonios hacen una constante referencia a estancias en Barcelona con personas relacionadas con el movimiento de renovación pedagógica Rosa Sensat ${ }^{5}$, destacando lo positivo de las mismas y el conocimiento de los métodos de Montessori, Decroly, Freire o Freinet. El recurso a estos autores fue comúnmente aceptado ya que su formación pedagógica en las Escuelas de Magisterio de la época no desarrollaba ninguna técnica como la imprenta, el aprendizaje dialógico, los centros de interés, etc. En ese contexto todos estos recursos didácticos resultaban verdaderas novedades pedagógicas. Se aprecia que la mayoría de los testimonios demanda una formación teórica y práctica en estas innovaciones que circulaban entre el magisterio renovador. El contacto con María Antonia Canals o Marta Mata es destacado por algunos testimonios por su aportación al cambio en la formación inicial (Testimonio IB; Maestra, Escuela pública).

En cuanto a la valoración global de la formación inicial recibida y su significado de cara a la renovación pedagógica, hay que resaltar opiniones muy positivas, como la de Neli Zaitegi, que destaca la pasión por la profesión y el gusto por el aprendizaje, por el conocimiento, por aprender a aprender, aunque no deje de criticar que la formación inicial debe ser otra cosa y contribuir a que la misma ayude al alumnado a transformar el mundo. En otros casos se demanda que esos estudios sean de más alto nivel y basados más en teorías contemporáneas, tanto psicológicas como pedagógicas. La visión crítica también muestra hasta qué punto la

\footnotetext{
${ }^{5}$ La Asociación de Maestros Rosa Sensat es un movimiento de renovación pedagógica creada en 1965 y que, desde ese momento, trabaja por la mejora de la educación a través de cursos, talleres y seminarios para la formación de los maestros y maestras. Toma su nombre de una maestra y pedagoga que nación en 1873 y falleció en 1961 que destacó por innovar la formación pedagógica de los maestros y maestras, aplicando las innovaciones que se estaban produciendo en Europa. Esta asociación es una referencia obligada en temas de renovación pedagaógica. Se puede obtener mas información en la Web de la asociación http://www2.rosasensat.org/. Recuperado en: 28 de octubre de 2018 .
} 
formación estaba basada en conocimientos tradicionales, sin que se introdujeran conocimientos más innovadores (Testimonio BL, Maestra, Ikastola).

\section{El ejercicio profesional y la compartición de la renovación pedagógica}

Una de las características que más señalan nuestros testimonios al recordar sus primeros años de ejercicio profesional es la reflexión sobre la práctica que estaban desarrollando, pues, de alguna manera, pone en evidencia la formación recibida y los contrastes con la realidad: "la práctica era nuestro punto de partida y luego hacíamos una reflexión sobre la misma y eso para mí era mucho más enriquecedor que hacer lo contrario" (Testimonio JMA; Maestro, Ikastola). En este contexto de reflexión y, sobre todo, cuando las necesidades eran compartidas, la creación de contactos o búsqueda de compañeros, con ideas y prácticas afines, era un elemento importante para orientar los objetivos innovadores. En este sentido, la creación de una "red de compañeros" que ejercían la profesión docente se veía como una necesidad ineludible: "Yo creo que el grupo de profesores es importante, porque un solo profesor no hace nada [...]. Nosotros tuvimos eso que significó una gran ayuda" (Testimonio JRE; Experto, Ikastola).

Pero esta reflexión y forma de actuar no solamente se refería a la situación del euskara, sino a las carencias que apreciaban en sus primeros pasos en la profesión docente. De manera que una de las carencias más manifiestas era la relativa a las necesidades de formación. La asistencia a cursos para adquirir competencias concretas, sobre todo relacionadas con la función didáctica, era un elemento importante en esos primeros años de ejercicio profesional. Esos cursos estaban muy relacionados con cuestiones concretas que se podían abastecer por medio de los recién creados Centros de Apoyo y Recursos (CAR) en los años 80, pero también teniendo acceso a una formación basada en metodologías renovadoras. Los cursos más frecuentes eran los relacionados con la didáctica de la lengua y la didáctica en general. Así, “íbamos a Cataluña a hacer cursos de Rosa Sensat, ellos venían una vez o dos al año al Colegio Corazón de María de Donostia y hacíamos ese intercambio, trabajando las metodologías que entonces estaban de moda" (Testimonio PB; Maestra, Escuela Pública).

También muchos de estos cursos estaban ligados con la creación de material didáctico, la organización de seminarios, cursos, visitas a escuelas, etc. De ahí que la necesidad de elaborar material fuera un elemento de la mayor importancia, primero porque el material existente no era útil para las necesidades de las ikastolas, inmersas en proceso de renovación donde los libros de texto tenían un interés limitado, y, por otra, porque el material tenía que estar redactado en euskara. En este sentido uno de los autores que más temprano comenzó a crear este tipo de 
material fue José Ramón Etxebarria, quien, en 1971, publicó el texto "Haizeak, euria eta klima" [Los vientos, la lluvia y el clima], iniciando, así, un camino dentro de la pedagogía, a pesar de que su formación inicial era del área de la física. Un testimonio nos recuerda que el libro de fichas de matemáticas de Feli Etxeberria fue el primer libro que utilizaron algunas maestras para su enseñanza y que el material creado por ellos mismos era de mayor utilidad, recordando que los sábados por la mañana se juntaban y se pasaban las programaciones (Testimonio MI; Maestra, Ikastola).

Por lo tanto, la falta de material supuso la implicación del propio profesorado en su creación. Se trataba de material que, en muchos casos, se elaboraba de un día para otro, compartiendo las tareas entre los propios docentes: "Nos juntábamos y preguntábamos entre nosotras ‘Cómo haces tú esto?' 'Pues no sé, yo de esta manera” y en el tren comenzábamos a preparar el material del día siguiente" (Testimonio PB; Maestra, Escuela Pública). En el mismo sentido se expresa otro testimonio: "Nosotros preparábamos los materiales -más tarde hubo personal liberado para ello - y los profesores nos reuníamos por niveles una vez al mes y presentábamos la utilización del material que creábamos, este material se habrá tirado, pero hicimos mucho material" (Testimonio AS; Maestra, Ikastola). En algunos casos, debido a la inexistencia de material, se utilizaban referencias de otras editoriales para la creación de estos proyectos educativos o se creaban proyectos específicos de temas correspondientes a ciertos niveles de EGB y se amoldaban a las necesidades concretas de la ikastola concreta en la que se iban a aplicar.

Desde luego, uno de los aspectos más relevantes en cuanto a las influencias pedagógicas es la consideración de los autores que fueron fuente de información para orientar la práctica educativa. A través de diversos cursos se accedió al conocimiento de los autores antes citados y otros, como se ha podido comprobar por las actividades realizadas por el colectivo de renovación pedagógica Adarra (BIOTA; ZABALETA, 2018), y estos mismos autores se convierten en la referencia primordial. En este sentido, los testimonios son conscientes del impacto que les causaron en su forma de ver la escuela y, sobre todo, los modelos pedagógicos que transmitían. Del mismo modo se abrieron caminos, no solamente a la euskaldunización del proceso escolar, sino también a los nuevos planteamientos pedagógicos y metodologías innovadoras. La asistencia a los cursos de verano organizados por Adarra es una constante en muchos de los testimonios recogidos, de todos modos, algunos valoran la formación impartida por estos colectivos como "muy reducida" ya que, aunque su idea es llegar al número mayor de gente, "no es formación permanente, pues no se da durante todo el curso. Es una formación que se da, sobre todo, en verano" (Testimonio IE; Maestro, Ikastola). 
No podemos olvidar que en los años 80 ya se había puesto en marcha por parte de la Consejería de Educación del Gobierno Vasco la Dirección de Innovación Pedagógica que ofertaría una serie de cursos de formación con unas líneas estratégicas marcadas por las políticas y necesidades educativas del momento. En este sentido cabe destacar la preocupación por introducir en las escuelas las Nuevas Tecnologías de la Información y Comunicación y otro tipo de planteamientos relativos a la inclusión educativa, además del proceso de euskaldunización, tanto del profesorado como del sistema educativo.

Sin embargo, hay que resaltar que, más allá de las teorías que sustentaban estos pedagogos y pedagogas, lo importante a rescatar de los mismos eran los aspectos metodológicos. Es decir, cómo enseñar de una manera intuitiva y práctica las diversas disciplinas que conformaban el curriculum. No obstante, algunos testimonios también ponen de manifiesto las influencias recibidas de compañeros más cercanos como es el caso de Imanol Urbieta (Testimonio PB; Maestra, Escuela Pública), Imanol Añón o Kepa Martínez de Lagos, que trabajó en el ámbito de las ciencias sociales (Testimonio JRE; Experto, Ikastola).

La visión de uno mismo como maestro/a cuando se está ejerciendo esta profesión también es un elemento a resaltar. De manera que, según los testimonios, los propios docentes se hacen un autorretrato en el que se refleja una imagen muy positiva de ellos mismos y del nivel de entusiasmo manifestado. Son escasos los testimonios que reflexionen sobre ese momento de una manera negativa. En primer lugar, porque el sentirse partícipes de un proceso compartido en el que el cambio de modelo de escuela y la euskaldunización del alumnado eran elementos dinamizadores de la actividad y, en segundo lugar, porque sentían que colaboraban así a un cambio social y personal. Las preocupaciones no solo se refieren a cuestiones de tipo contextual, sino a prácticas relacionadas con la actividad profesional: "Siempre me he preocupado de aprender, de metodologías, en el aspecto del euskera, por el bilingüismo, por el aspecto de la lingüística... y bueno, eso. Cuando me ha tocado la parte de la evaluación, ¡no me he quedado quieta!” (Testimonio AS; Maestra, Ikastola). En otros casos el motor de esta actividad como herramienta para superar los obstáculos era la propia intuición en la actividad docente (Testimonio JMA; Maestro, Ikastola).

\section{La demanda continua de formación permanente}

Una de las características de la renovación pedagógica es, sin duda, la formación permanente del profesorado. Se trata de una premisa básica para que ese proceso no se quede estancado, paralizando así las posibilidades de innovación en el ejercicio profesional docente. 
Como era de esperar, las opiniones vertidas por nuestros testimonios se reafirman en muchas de las actividades que realizaban en su ejercicio profesional. Por lo tanto, el núcleo de cuestiones que se planteaban giraba alrededor de la opinión del profesorado, de la participación en sus actividades, de las asociaciones de renovación pedagógica y de la participación de los propios testimonios (MONTERO, 2006, IMBERNON, 2014, MARTÍNEZ BONAFÉ, 2014). Sin duda, la formación permanente en el País Vasco ha seguido ritmos parecidos a la llevada a cabo en el resto de las Comunidades Autónomas por lo que respecta a la creación de instituciones, partiendo del plan general que se elaboró en 1989, aunque uno de los elementos más importantes ha sido la distribución de los recursos de formación permanente por zonas a partir de la creación de los Centros de Orientación Pedagógica (COP) (BARCELÓ, 1996).

En general, la opinión sobre la formación permanente y su necesidad tiene tres núcleos fundamentales: 1) La necesaria autoformación, a través, fundamentalmente, de la lectura; 2) La colaboración entre docentes y 3) La participación en cursos, seminarios, cursos de verano, etc. que se veían como fundamentales. No obstante, se puede observar que algún testimonio entiende que la formación permanente no es una actividad que se considere voluntaria, sino que más bien es un derecho y, al mismo tiempo, una obligación: “yo la veo absolutamente necesaria y la formación, para mí, es obligación y derecho [...]. La formación debería tener una doble dirección, a veces de arriba abajo y otras de abajo arriba" (Testimonio BL; Maestra de ikastolas). Esta afirmación muestra el verdadero sentido que, en la práctica, se llevaba a cabo. Se podría decir que este tipo de colaboración entre docentes requiere un entusiasmo y un saber compartir los proyectos que se ajusten a las necesidades prácticas que se viven en la escuela.

Por otra parte, la participación en cursos y seminarios se valora como la manera más óptima de definir la formación permanente, incluso cuando el profesorado parte de su propia formación: “Toda formación te pide más formación. Lees algo, lo pones en práctica, no funciona, esto te pide entonces otra lectura, discutir, etc... entonces en verano íbamos a cualquier curso, tanto en Cataluña con Rosa Sensat como aquí, los que organizaba Adarra, aunque la mayoría eran en castellano. Para entonces ya existía Irakasleen Elkartea ${ }^{6}$, especialmente en Gipuzkoa, la mayoría de los asistentes profesores de ikastolas, íbamos alrededor de 500, organizábamos cursos de matemáticas, de lectura y similares" (Testimonio JMA; Maestro, Ikastola). Por lo tanto, como se puede apreciar por los testimonios, estos cursos estaban organizados tanto por el colectivo como por Irakasleen Elkartea, la Federación de Ikastolas o los Centros de Profesores, en general a través de cursos de verano. En este sentido,

\footnotetext{
${ }^{6}$ Asociación de profesores.
} 
como algún testimonio manifiesta en los primeros años de la década de los 80 , podemos decir que este tipo de asociaciones, organismos e instituciones era como un puzle con piezas que se complementaban y, seguramente, dirigidos a segmentos del profesorado que compartían un mismo modelo de escuela, aunque también es cierto que algunos testimonios asistían indiferentemente a cursos organizados al margen de la institución o colectivo que los organizaba.

Lo interesante de este modelo de formación es que, de forma inapreciable, se pasa de los cursos informales organizados por diferentes asociaciones a los cursos y seminarios organizados desde la Consejería de Educación: "los COP han ayudado mucho al proceso de renovación pedagógica" (Testimonio AS; Maestra, Ikastola). En el mismo sentido se expresa otro testimonio cuando se refiere a las actividades de la Federación y de Adarra y de sus cursos del mes de julio, señalando que más tarde "llegaron los cursos de los COP del Gobierno Vasco que se realizaban a lo largo del curso. Estábamos obligados y luego ya se plantearon otros cursos en el claustro" (Testimonio IB; Maestra, Escuela pública). Como se puede apreciar hay un hilo de continuidad entre los cursos no formales y la institucionalización llevada a cabo por la administración educativa del momento. En estos cursos la voz de los claustros de las escuelas era muy valorada, en cuanto que sus propuestas eran atendidas por la institución. También hay que tener en cuenta que los centros regidos por las órdenes y congregaciones religiosas también tenían su propio camino para la formación permanente. Así el testimonio de Joxe Erzibengoa nos indica el éxito obtenido por el libro "La formación permanente del Hermano en la actualidad" en el que muestra desde el punto de vista de La Salle las nuevas aportaciones de la pedagogía y de la psicología, lo que significó para algunos una nueva forma de ver la escuela "Ese libro yo lo tomé, como otros toman la Biblia" (Testimonio JE; Maestro, Escuela privada), algo similar ocurría en el ámbito de los jesuitas a través de los cursos de formación de la Universidad de Deusto o la referencia a Imanol Añón, que era el responsable de todos los centros de los jesuitas en el País Vasco y que fomentó la introducción del euskara y su metodología de enseñanza (Testimonio JRE; Experto, Ikastola).

A través de esta formación permanente interesa también detectar qué modelos formativos habían conocido y de qué manera pudiera haber servido para orientar la práctica docente. Esto supone superar un conflicto que algún testimonio pone de manifiesto, como es la imposibilidad de llevar muchas veces a la práctica aquella formación teórica recibida. Esta situación es relevante en cuanto podría significar una fuente de conflictos para el profesorado, ya que, como modelo teórico, puede funcionar muy bien, pero la realidad obliga a una adecuación de las teorías aprendidas. Las frustraciones por esta imposibilidad de poner en 
práctica los modelos obligan a remodelar o adaptar los aspectos que la realidad pudiese asumir y la falta de un diseño global de la formación que muchas veces llevaba al profesorado a hacer cursos por "moda" y que, al cabo de poco tiempo, se descartaba lo que hacía poco tiempo era fundamental.

Lo que sí queda evidente en todo este proceso es que el modelo de escuela que tienen en mente los maestros/as innovadores es compartido ya que la renovación pedagógica y las nuevas experiencias que están surgiendo alrededor del movimiento de ikastolas se están desarrollando conjuntamente: se trata de una escuela vasca entendida como "servicio público", o pública en el sentido tradicional. En la década de los 80 se produce un salto cualitativo, sobre todo en el ámbito de la formación e institucionalización de los proyectos que unen la renovación pedagógica y el proceso de euskaldunización en las ikastolas con un proceso de normalización interna, que repercutirá en los modos de enfocar la formación permanente. Algunas veces más centrada en los componentes organizativos y de dirección que en los meramente pedagógicos, pues también se trata de un modelo de autoorganización social, autónoma, independiente, autogestionada, que, por sí mismo, es también un modelo renovador.

Por lo tanto, por lo que se refiere a la actualización docente por parte del profesorado se aprecia que la mayoría de testimonios se refiere a los mismos centros u organizaciones implicadas en la renovación pedagógica, sea los ya tantas veces citado Adarra y sus jornadas pedagógicas, los cursos de verano, incluidos los de la Udako Euskal Unibertsitatea ${ }^{7}$, los grupos de trabajo alrededor de la renovación e, incluso, otros cursos organizados por movimientos de renovación pedagógica fuera del País Vasco. No obstante, en algunos casos se aprecia, con el paso del tiempo, cierto desánimo en este tipo de actividades: "Creo que hemos perdido esa dedicación inicial, esas ganas de investigar que teníamos" (Testimonio IB; Maestra, Escuela pública). En otros casos no se trata de desánimo, sino más bien de apreciar cierta inutilidad en los contenidos de los cursos en los que la participación del profesorado es más pasiva y que dejan de tener interés, valorando mucho más los proyectos compartidos con los compañeros del claustro. Con respecto al euskara, este tipo de renovación demandaba una mayor implicación por cuanto al "euskara y a la cultura vasca se le han puesto muchos obstáculos durante muchos años y para que tuvieran su lugar en la cotidianeidad había que hacer un trabajo especial" (Testimonio MI; Maestra, Ikastola).

Esta actualización docente también pone de manifiesto las influencias en la formación permanente y en la participación por parte de los maestros y maestras entre la década de los 80

\footnotetext{
${ }^{7}$ Universidad Vasca de Verano
} 
y de los 90, de manera que los lugares comunes y los centros de formación que hemos ido citando aparecen frecuentemente, así se aprecia que estos maestros renovadores hacían una especie de exploración por el terreno de la renovación a fin de ir alimentando sus intereses de reformar la escuela, innovar en las metodologías y compartir un tipo de escuela en la cual se privilegiasen los aspectos de una enseñanza pública y, por lo tanto, estatal o bien como un servicio público de carácter social y, por supuesto, donde estuviese presente el euskara. Es decir, la cita continua de asistencia a cursos organizados por el movimiento de renovación pedagógica tanto en el País Vasco como en otros lugares del Estado, resultará asidua, así como los cursos organizados por la Federación de Ikastolas, Irakasleen Elkartea, Adarra o la Udako Euskal Unibertsitatea. Por lo tanto, el marco de opciones era amplio y significó, en todo momento, una apertura a nuevos enfoques, la mayoría de ellos con un sentido muy práctico, encaminados a mejorar la calidad de las escuelas. Junto con ello el trabajo en grupo también parecía que era otro elemento que influenció la formación permanente y que es muy apreciado por parte de los maestros y maestras que estaban motivados a participar.

Lo que sí queda evidente en todo este proceso es que, al estar desarrollándose conjuntamente, la renovación pedagógica y las nuevas experiencias que están surgiendo alrededor del movimiento de ikastolas, se aprecia que el modelo de escuela que tienen en mente los maestros y maestras innovadores es compartido. En la década de los 80 se produce un salto cualitativo, sobre todo, en el ámbito de la formación e institucionalización de los proyectos que unen la renovación pedagógica y el proceso de euskaldunización en las ikastolas con un proceso de normalización interna que repercutirá en los modos de enfocar la formación permanente. Algunas veces más centrada en los componentes organizativos y de dirección que los meramente pedagógicos, pues también se trata de un modelo de autoorganización social, autónoma, independiente, autogestionada, que también es un modelo renovador por sí mismo.

\section{CONCLUSIONES}

Las generaciones, desde el punto de vista profesional, nos han permitido detectar las características individuales, los proyectos y la participación de los testimonios en un movimiento de renovación pedagógica donde el contexto político, social y cultural era determinante, tal y como hemos recogido a través de las voces de los maestros/as. La renovación pedagógica ha actuado como un motor que permitía dar sentido a la vida profesional de muchos maestros/as en el País Vasco. Se trataba no solamente de una opción por cambiar el tipo de escuela que criticaban, sino de proyectar un nuevo modelo que pudiese estar en 
consonancia con los cambios políticos y educativos que se estaban produciendo. Asimismo, el euskara era un elemento que coadyuvaba, en el sentido vital que muchos testimonios experimentaban, de manera que se entendía, en muchos casos, que la renovación pedagógica no podría llevarse a cabo si, de manera simultánea, no se desarrollaban actividades que pudiesen euskaldunizar al profesorado, al alumnado y, en definitiva, al tipo de escuela que se estaba construyendo (ikastolas o escuela pública). Asimismo, hemos resaltado el valor patrimonial que tienen las voces de los maestros y maestras protagonistas en un momento clave de su desarrollo profesional. En este sentido, resulta relevante que el guion museográfico que contiene el relato de la historia de la educación en el País Vasco, contenga las voces de los protagonistas. De esta manera, el público visitante toma conciencia del valor histórico y patrimonial que tienen los protagonistas en los fenómenos educativos, en este caso, los maestros y maestras y la renovación pedagógica.

La renovación pedagógica, en cuanto al relato que hacen de la formación inicial nuestros testimonios, refiriéndose tanto al tipo de escuela conocido en su propia experiencia como alumno, como al recibido en las instituciones de formación de profesorado, vendría caracterizada por tratarse de una formación autoritaria y de modelo "franquista". La elección de esta profesión, en la mayoría de los casos, venía determinada por una doble preocupación: la situación política que se estaba viviendo y la pérdida del euskara. En este sentido, los testimonios construyen un relato cronológico de la situación política muy cercano. De la misma manera, la lucha por el euskara era un elemento determinante para optar por la enseñanza, vista la situación de recuperación de la lengua en esos años.

En cuanto a la actividad profesional, hay que destacar el tipo de actividades que se desarrollaban, donde el compartir las experiencias con el resto de maestros/as con los que ejercían la profesión resultaba fundamental. De hecho, la opinión más valorada con respecto a la actividad profesional es el poder compartir un proyecto de renovación pedagógica y de recuperación del euskara. Asimismo, los testimonios destacan un elemento importante en cuanto a la formación en el ámbito del ejercicio de la profesión, como es la adquisición de conocimientos y estrategias para mejorar sus metodologías didácticas. Asimismo, la creación de material por los propios profesores o el uso de material creado por otros compañeros era una práctica extendida. En el ejercicio profesional quedan patentes las diferencias de opinión sobre el tipo de escuela, sea pública, privada religiosa o ikastola, de manera que parece apreciarse una cierta coexistencia y respeto, sobre todo, porque en muchos casos se compartía un mismo horizonte sobre el tipo de escuela que se deseaba, prioritariamente como un servicio público y vasca. Todo ello con muchos matices en función del lugar en el cual se ejerciese la profesión. 
Asimismo, los modelos pedagógicos y didácticos que aparecen en los testimonios son comunes, de manera que autores como Freinet, Montessori, Decroly, Freire, etc. son guías que orientan las reformas que se están llevando a cabo, aunque también se señala la influencia de compañeros que desarrollan, en su práctica educativa, proyectos innovadores. Hay que resaltar que, aunque estos autores fueron innovadores tiempo atrás de ese momento, lo cierto es que, a la vista de que la escuela en ese momento seguía unos patrones tradiciones, cualquier innovación introducida por ellos resultaba, en sí innovadora.

La formación permanente es destacable pues muestra el momento de preocupación, tanto de las instituciones de administración educativa como de los propios maestros/as. La necesidad de esta formación permanente es una afirmación unánime de todos los testimonios, de manera que la participación en cursos, seminarios, etc. no se veía como una opción, sino como una obligación y derecho. La asistencia constante a curso de asociaciones y organismos como Adarra, Irakasleen Elkartea, Gordailu, Udako Euskal Unibertsitatea, además de a los de Rosa Sensat en Cataluña o los congresos de los Movimientos de Renovación Pedagógica, conforman una red de formación permanente, sin dejar de lado la formación impartida a través de los Centros de Orientación Pedagógica organizados desde la Consejera de Educación. Esta actualización docente se manifiesta en el hecho de compartir metodologías existosas con otros compañeros, por lo cual se vuelve a poner de manifiesto que compartir un proyecto entre compañeros era un elemento clave de la renovación pedagógica.

A la vista de estas características de esta generación de maestros/as, se podría plantear la existencia de dos modelos de profesorado, según el objeto que se quiera destacar dentro de la renovación pedagógica; por una parte, un profesorado pedagogicista, muy preocupado por cambiar la escuela desde dentro, por implementar teorías pedagógicas innovadoras, por introducir en el aula nuevos planteamientos didácticos, etc.; y otro, un profesorado contextualista, con un mayor interés en la sociedad, ya que la escuela se entendía como una extensión del tipo de escuela promovida por la sociedad del momento. De todas maneras, tanto en un tipo de profesorado como en otro, el euskara actúa como un detonante de la renovación pedagógica. Se podría afirmar que son maestros/as renovadores si con ello se consigue que el euskara se recupere y se extienda, no solamente para su uso en la escuela, sino también en toda la sociedad, ya que en ese momento la construcción nacional de Euskal Herria tenía que pasar inevitablemente por la presencia del euskara en el sistema educativo y fuera de él, ya que la lengua es su mayor símbolo identitario. La mayoría de estos maestros y maestras eran conscientes de que la escuela era el mejor vehículo para la pervivencia del euskara y que, por lo tanto, el magisterio tenía la responsabilidad de recuperar, difundir y asegurar la lengua 
propia. En esa época un lema comúnmente aceptado era que Euskal Herria no podía existir sin el euskara.

\section{BIBLIOGRAFÍA}

BARCELÓ, Fermín. La formación permanente en la Comunidad Autónoma del País Vasco. Em: ANGULO, Luis Miguel. La formación permanente del profesorado en el nuevo sistema educativo en España. Barcelona: Oikos-Tau, 1996. p. 203-225.

BIOTA, Itsaso; ZABALETA, Iñaki. ADARRA: la renovación pedagógica al servicio de la escuela pública vasca. En: DÁVILA, Paulí (coord..). El profesorado y la renovación pedagógica en el País Vasco. Madrid: Editorial Delta, 2018. p. 85-130.

BOLÍVAR, Antonio. Las historias de vida del profesorado: posibilidades y peligros, ConCiencia Social, n. 9, p. 59-69, 2005.

Las historias de vida del profesorado: voces y contextos, RMIE Revista mexicana de investigación educativa, v. 19, n. 69, 2014.

; DOMINGO, Jesús; FERNÁNDEZ, Manuel. La investigación biográfico-narrativa en educación. Madrid: Editorial La Muralla, 2001.

CABALLERO, Manuela; BAIGORRI, Artemio. ¿Es operativo el concepto de generación? Aposta. Revista de Ciencias Sociales, n. 56, 2013. Accesible en: http://www.apostadigital.com/revistav3/hemeroteca/mcg1.pdf $>$. Recuperado en: 27 de agosto de 2018.

DÁVILA, Paulí. La renovación pedagógica en el País Vasco: segunda mitad del siglo XX, Sarmiento, n. 9, p. 85-104, 2005.

(Coord.). El profesorado y la renovación pedagógica en el País Vasco. Madrid: Editorial Delta, 2018.

DELGADO, Ander. Gordailu y la renovación pedagógica en el País Vasco. Política y educación en el tardofranquismo. Revista Aula, n, 22, p. 189-203, 2016.

EUSKAL HERRIKO IKASTOLAK; EUSKALTZAINDIA. El movimiento de las ikastolas un pueblo en marcha. El Modelo Ikastola 1960-2010. Bilbao: Euskaltzaindia, 2011.

GIL, Fernando. Sociología del Profesorado. Barcelona, Ariel, 1996.

GOMEZ, Luis Alberto; ESCUDERO, Juan Manuel (ed.). La formación del profesorado y la mejora de la educación. Barcelona: Octaedro, 2006.

IMBERNÓN, Francisco. La formación permanente 40 años después: de la ilusión a los recortes, Cuadernos de Pedagogía, n. 451, p. 56-59, 2014. 

2017.

Ser docente en una sociedad compleja. La difícil tarea de enseñar. Barcelona: Graó,

(Coord.). Vivencias de maestros y maestras. Compartir desde la práctica educativa. Barcelona: Graó Ediciones, 2005.

LASKY, Sue. A sociocultural approach to understanding teacher identity, agency and professional vulnerability in a context of secondary school reform, Teaching and Teacher Education, v. 21, n. 8, p. 899-916, 2005.

LEITE, Analia. Historias de vidas docentes: recuperando, reconstruyendo y resignificando identidades, Praxis educativa, v. XVI, n. 1, p. 13-21, 2012.

LOPES, Amelia; HERNÁNDEZ, Fernando; SANCHO, Juana María, RIVAS, Jose Ignacio. Histórias de Vida em Educação: a construção do conhecimento a partir de histórias de vida. Barcelona: Universitat de Barcelona, 2013.

MARÍAS, Julián. El método histórico de las generaciones. Madrid: Revista de Occidente, 1949.

MARTINEZ BONAFÉ, Jaume. Utopías pedagógicas: logros y frustraciones, Cuadernos de Pedagogía, n. 451, p. 44-47, 2014.

MONTERO, María Luisa. Las instituciones de la formación permanente, los formadores y las políticas de formación en el Estado de las Autonomías. En: GOMEZ, Luis Alberto y ESCUDERO, Juan Manuel. La formación del profesorado y la mejora de la educación: políticas y prácticas. Barcelona, Octaedro, 2006. p. 155-196.

NÓVOA, Antonio (coord.). Vidas de professores. Porto: Porto Editora, 1992.

ORTEGA, Félix. Los estudios de magisterio en España: análisis de una década. En: GRAÑERAS, Montserrat; LAMELAS, Ricardo; CERDÁN, Jesús. La investigación sobre profesorado (II) 1993-1997, Madrid: CIDE, 1999. p. 117-136.

PERICACHO, Francisco Javier. Pasado y presente de la renovación pedagógica en España (de finales del siglo XIX a nuestros días). Un recorrido a través de escuelas emblemáticas. Revista Complutense e Educación, v. 25, n. 1, p. 47-67, 2014.

PINTO, José María. Los movimientos de renovación Pedagógica de la Comunidad de Madrid. Análisis de su influencia en el desarrollo profesional docente. Universidad de Valladolid (Tesis doctoral inédita), 2016.

PUJADAS, Juan José. EI método biográfico: historias de vida en ciencias sociales. Madrid: Centro de Investigaciones Sociológicas, 1992.

RIFÁ, Montserrat. Historias del currículum e historias de vida en el trabajo de investigación sobre la enseñanza de Ivor Goodson. Con-Ciencia Social, n. 9, p. 70-98, 2005.

SANCHO, Juana María. Historias de vida: el relato biográfico entre autoconocimiento y dar cuenta de la vida social, Praxis educativa, v. 18, n. 2. 2014. Accesible en: 
<http://www.redalyc.org/articulo.oa?id=153137900003>. Recuperado en: 27 de agosto de 2018.

(Coord.). Con voz Propia. Los cambios sociales y profesionales desde la experiencia de los docentes. Barcelona: Octaedro, 2011.

VIÑAO, Antonio. Las autobiografías, memorias y diarios como fuente histórico-educativo: tipologías y usos, Sarmiento, n. 3, p. 223-253, 1999.

Autobiografías, memorias y diarios de maestros y maestras en la España contemporánea, Cultura escrita y Sociedad, n. 8, p. 183-200, 2009.

ZUFIAURRE, Benjamín. ¿Se puede cambiar la educación sin contar con el profesorado? Barcelona: Octaedro, 2007. 